\title{
Solar Cell Emitters Fabricated by Flash Lamp Millisecond Annealing
}

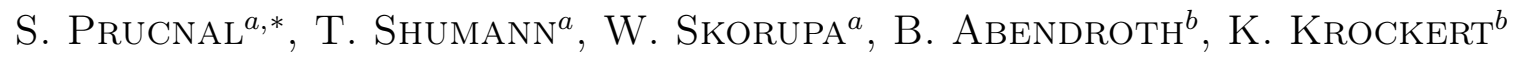 \\ AND H.J. MÖLLER ${ }^{b}$ \\ ${ }^{a}$ Institute of Ion Beam Physics and Materials Research, Helmholtz-Zentrum Dresden-Rossendorf \\ P.O. Box 510119, 01314 Dresden, Germany \\ ${ }^{b}$ Institute for Experimental Physics, TU Bergakademie Freiberg, Leipziger Str. 23, 09599 Freiberg, Germany

\begin{abstract}
Phosphorus ion implantation was used for the emitter formation in mono- and multicrystalline silicon solar
\end{abstract} \\ cells. After ion implantation the silicon is strongly disordered or amorphous within the ion range. Therefore \\ subsequent annealing is required to remove the implantation damage and activate the doping element. Flash-lamp \\ annealing offers here an alternative route for the emitter formation at overall low thermal budget. During \\ flash-lamp annealing, only the wafer surface is heated homogeneously to very high temperatures at ms time \\ scales, resulting in annealing of the implantation damage and electrical activation of phosphorus. However, \\ variation of the pulse time also allows to modify the degree of annealing of the bulk region to some extent \\ as well, which can have an influence on the gettering behaviour of metallic bulk impurities. The $\mu$-Raman \\ spectroscopy showed that the silicon surface is amorphous after ion implantation. It could be demonstrated that \\ flash-lamp annealing at $800^{\circ} \mathrm{C}$ for $20 \mathrm{~ms}$ even without preheating is sufficient to recrystallize implanted silicon. \\ The highest carrier concentration and efficiency as well as the lowest resistivity were obtained after annealing \\ at $1200{ }^{\circ} \mathrm{C}$ for $20 \mathrm{~ms}$ both for mono- and multicrystalline silicon wafers. Photoluminescence results point to- \\ wards P-cluster formation at high annealing temperatures which affects metal impurity gettering within the emitter.
}

PACS: 88.40.jj, 78.55.-m, 78.30.Am, 78.55.Ap

\section{Introduction}

Cost reduction is the overall goal in the further development of solar cell technologies. Processing solar cells at lower temperatures helps to reduce the energy cost and in thin film technologies may also facilitate the use of less temperature stable substrates such as normal glass or polymer foils. Recently, multicrystalline silicon (mc-Si) solar cells made of either bulk material or thin films have attracted considerable attention because of their high stability against light soaking. Poly-Si films are very important for silicon-gate metal oxide semiconductor (MOS) integrated circuits, charge-coupled devices, solar cells, thin-film transistors (TFTs), and various other applications [1-4].

In the case of low quality mc-Si (containing relatively high concentration of metal impurities) low temperature process is desirable for solar cells fabrication. In order to avoid diffusion of metals into charge space region of the $p-n$ junction, the bulk of the mc-Si material should not exceed $500{ }^{\circ} \mathrm{C}$. Due to metal impurities the conventional solar cell processing of the emitter fabrication by indiffusion of the doping element is not recommended.

* corresponding author; e-mail: s.prucnal@fzd.de
Therefore the doping elements are introduced into silicon by either conventional ion implantation or plasma immersion ion implantation (PIII) [5].

Independent of the implantation techniques, after ion irradiation silicon is strongly disordered or amorphous within the ion range. In order to recrystallize silicon and activate dopants, the wafers have to be annealed in such a way that only the surface (up to $100 \mathrm{~nm}$ ) will be heated which makes furnace annealing (FA) or rapid thermal annealing (RTA) techniques useless.

One alternative method to FA or RTA is excimer laser annealing (ELA), successfully used for mc-Si film formation [6]. During ELA silicon surface is heated to its melting point within tens of nanoseconds which allows us to recrystallize silicon and activate dopants without heating the bulk material or glass substrate. The main disadvantage of the ELA techniques is achieving a non-uniform recrystallization on the large substrates and very high costs of equipments which eliminates ELA from solar cells production.

In the present work a new technique will be presented and explored, which allows to activate implanted elements by short time light pulse annealing. The flash-lamp annealing (FLA) systems are successfully used for silicon recrystallization in many laboratories $[1,7-9]$. In principle, there is no sample size limitation for the FLA 
systems but producing a homogeneously annealed big area wafer (at least an eight inch one) is not trivial. The phosphorus implanted and annealed silicon wafers were characterised by means of $\mu$-Raman spectroscopy, photoluminescence, and four-point probe resistor measurements. Influence of the annealing time and temperature on the optical, microstructural and electrical properties on the c-Si and mc-Si based solar cell emitters are presented.

\section{Experimental part}

The $p-n$ junctions were formed in mono- and multicrystalline $p$-type silicon wafers by phosphorus implantation and milliseconds range FLA. Phosphorus was implanted by a conventional ion beam implanter with the fluence of $2 \times 10^{15} / \mathrm{cm}^{2}$ and an energy of $120 \mathrm{keV}$. A $120 \mathrm{~nm}$ surface $\mathrm{SiO}_{2}$ layer was deposited by plasma enhanced chemical vapour deposition, resulting in a projected range of the implanted phosphorus of about $30 \mathrm{~nm}$ from the silicon surface. The depth profile of the implanted P was calculated using the SRIM 2003 code. After implantation the samples were annealed at three different temperatures 800,1000 and $1200^{\circ} \mathrm{C}$ for $20 \mathrm{~ms}$ without or with preheating at 400 or $600^{\circ} \mathrm{C}$ for $3 \mathrm{~min}$. Structural and optical properties of silicon wafers were investigated by means of $\mu$-Raman spectroscopy (RS) and photoluminescence (PL). The $\mu$-Raman spectra were recorded at room temperature in the backscattering geometry in the range 150 to $600 \mathrm{~cm}^{-1}$ using $532 \mathrm{~nm}$ YAG laser. The PL spectra were recorded at room temperature using the Jobin Yvon Triax 550 monochromator and a cooled InGaAs detector. For the sample excitation during PL measurements the $532 \mathrm{~nm}$ laser light with a power of 20 or $200 \mathrm{~mW}$ was applied. For the sheet resistance (SR) measurements the four-point probe resistor system (CMT-SR 3000 Advanced Instrument Technology) was used.

\section{Results and discussion}

\subsection{Flash lamp annealing system}

Figure 1a shows the schema of the FLA system used in our experiments. It consists of two annealing systems: on backside - rapid thermal annealing made of halogen lamps and frontside - FLA containing twelve Xe lamps. Different gases can be introduced into the water cooled annealing chamber, allowing the annealing in different ambient gas. The maximum sample size which can be annealed in our system is limited due to the length of the halogen and Xe lamps (300 mm).

Halogen lamps are used for the backside preheating and the maximum temperature which can be obtained in our system is slightly above $950^{\circ} \mathrm{C}$ for silicon. The system provides possibility to anneal $300 \times 300 \mathrm{~mm}^{2}$ or $300 \mathrm{~mm}$ diameter silicon wafer. The maximum temperature during flash can easily reach the melting point of silicon and annealing time can vary from 0.8 up to $20 \mathrm{~ms}$ in a single
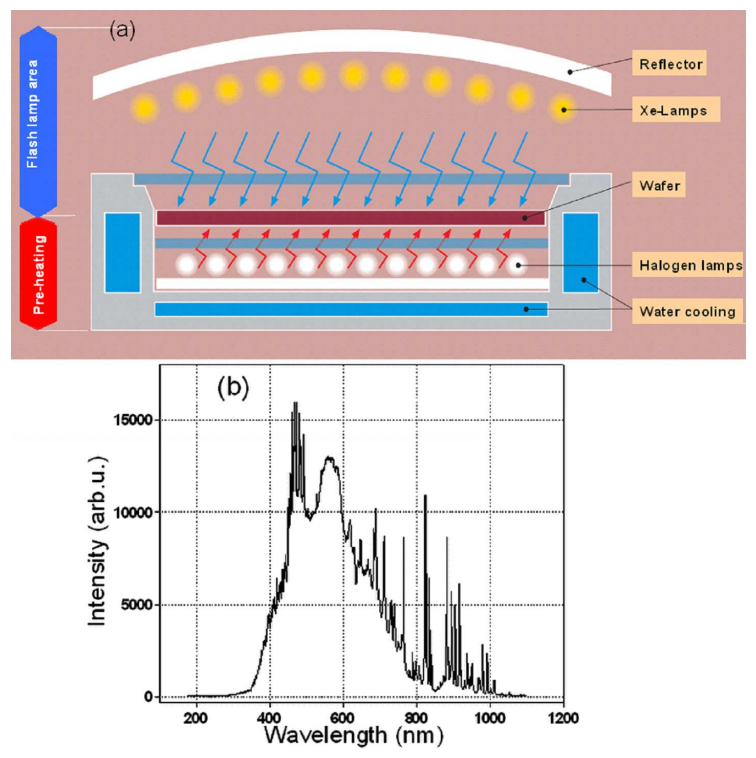

Fig. 1. Schematic illustration of the flash lamp annealing system (a) and Xe lamp spectrum (b).

shot with the temperature variation below $10 \%$ through the wafer area. In order to have a homogeneous temperature distribution throughout the wafer a special reflector was designed. The spectrum of Xe lamps is presented in Fig. 1b. The maximum intensity of Xe lamps used in our system falls in the range of 400 and $600 \mathrm{~nm}$. Therefore, for effective annealing, the material should have a high absorption coefficient in this region. The maximum energy which can be submitted to the sample during single $20 \mathrm{~ms}$ shot is $250 \mathrm{~kJ}$.

\subsection{Samples characterization}

The crystal quality of the silicon wafer after phosphorus implantation and flash lamp annealing was controlled by the Raman spectroscopy. The Raman spectra were recorded at room temperature within the wave number range from 200 to $600 \mathrm{~cm}^{-1}$ using a $532 \mathrm{~nm}$ laser. It is well known that ion implantation into a crystalline matrix introduces radiation defects and for high fluences amorphization of the implanted region takes place. Figures $2 \mathrm{a}$ and $\mathrm{b}$ show the Raman spectra obtained from c-Si and mc-Si wafers implanted with $\mathrm{P}^{+}$ions, respectively, and subsequently flash lamp annealed at 800, 1000 and $1200{ }^{\circ} \mathrm{C}$ for $20 \mathrm{~ms}$.

The Raman spectra of as-implanted and annealed samples at 400 and $600{ }^{\circ} \mathrm{C}$ for $3 \mathrm{~min}$ are presented in the inset of Fig. 2a. The as-implanted sample (see inset of Fig. 2a) shows a broad Raman band at around $460 \mathrm{~cm}^{-1}$ and a weak peak at around $520 \mathrm{~cm}^{-1}$. The band at $460 \mathrm{~cm}^{-1}$ corresponds to the amorphous silicon formed during ion implantation [10] while the peak at $520 \mathrm{~cm}^{-1}$ is a fingerprint of crystalline silicon substrate [11]. The projected range for phosphorus is around $30 \mathrm{~nm}$ from the silicon surface. Hence, taking the Gaussian distribution 


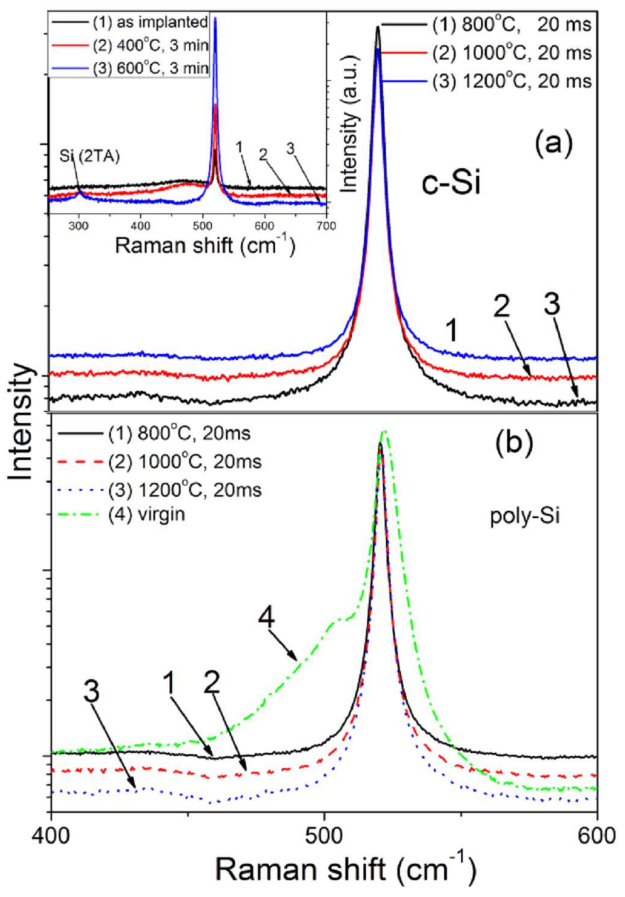

Fig. 2. $\mu$-Raman spectra obtained from $\mathrm{P}^{+}$implanted and annealed c-Si (a) and mc-Si (b) for $20 \mathrm{~ms}$ without preheating. Inset in (a) shows the results obtained from as-implanted c-Si samples and annealed at $400{ }^{\circ} \mathrm{C}$ or $600{ }^{\circ} \mathrm{C}$ for $3 \mathrm{~min}$.

of $\mathrm{P}$ atoms into account, the amorphization of the silicon layer due to ion implantation can reach maximum $80 \mathrm{~nm}$. Because the penetration depth of the green light (532 nm laser light used in the Raman measurements) in amorphous silicon is in the same range as implantation depth, the small peak visible at $520 \mathrm{~cm}^{-1}$ in the as-implanted sample is due to photons inelastically scattered from the crystalline silicon substrate. An increase of the annealing temperature of the silicon samples increases the intensity of the peak at $520 \mathrm{~cm}^{-1}$ due to recrystallization of the silicon layer damaged during ion implantation. According to the Raman measurements after $600{ }^{\circ} \mathrm{C}$ annealing for $3 \mathrm{~min}$ silicon is fully recrystallized. Moreover, after annealing $600{ }^{\circ} \mathrm{C}$ for $3 \mathrm{~min}$, an additional peak at $300 \mathrm{~cm}^{-1}$ which corresponds to the second-order two transverse acoustic phonon (2TA) scattering from crystalline Si was observed. This peak was visible in all flash annealed samples. As can be seen in Fig. 2a and b the flash lamp annealing for $20 \mathrm{~ms}$ even at such a low temperature as $800^{\circ} \mathrm{C}$ is sufficient to completely recrystallize the silicon.

The broad peak visible in Fig. $2 \mathrm{~b}$ at $505 \mathrm{~cm}^{-1}$ arises due to stress in the crystal grain. The effect of stress on the Raman spectra of Si originates from the lattice deformation, which leads to modified frequencies of the optical phonons [12]. The determination of stress in crystalline semiconductors by the Raman spectroscopy has been extensively investigated [13-15]. The stress $\sigma$ can be calcu- lated according to: $\sigma(\mathrm{GPa})=-0.2 \times \Delta \omega\left(\mathrm{cm}^{-1}\right)$, where $\Delta \omega$ is the frequency shift from $520 \mathrm{~cm}^{-1}$ to the measured silicon transverse optical (TO) Raman peak. Blue shift of the Raman signal results in compressive stress $(\sigma<0$ while tensile stress results in red shift and $\sigma$ is positive. In our case the stress is tensile and amounts to $\sigma=3 \mathrm{GPa}$. Flash lamp annealing of the virgin samples removes the stress from the sample completely (at least within the accuracy of the method). Moreover, the shift of the TO Raman peak $\left(520 \mathrm{~cm}^{-1}\right.$ line) after implantation and annealing was not detectable in our experiments and we have found that $800^{\circ} \mathrm{C}$ annealing for $20 \mathrm{~ms}$ is sufficient to activate phosphorus and recrystallized mc-Si.

Figure 3 shows room temperature photoluminescence spectra obtained from the implanted and annealed samples at 800,1000 or $1200^{\circ} \mathrm{C}$ for $20 \mathrm{~ms}$ c-Si with and without preheating. The low temperature annealed samples at 400 and $600{ }^{\circ} \mathrm{C}$ for $3 \mathrm{~min}$ are shown as well. During PL measurements green laser with $532 \mathrm{~nm}$ wavelength and $200 \mathrm{~mW}$ power was used with the incident angle $60^{\circ}$ to the normal. The broad peak at around $1140 \mathrm{~nm}$ corresponds to the band-to-band ( $\mathrm{B}-\mathrm{B})$ transition in silicon and the narrow one at $1064 \mathrm{~nm}$ is due to elastically scattered laser light from the sample. The side band of the $\mathrm{B}-\mathrm{B}$ with the maximum at around $1220 \mathrm{~nm}$ corresponds to the luminescence centers formed by phosphorus ions. The PL intensity strongly depends on the crystal quality of the silicon matrix. Such defects as: atom displacements, vacancies, dangling bonds etc. work as nonradiative channels for PL deexcitation and hence reducing the PL efficiency. It is worth noticing that many of these defects cannot be detected in the Raman spectrum but are easily visible in the PL measurements. After FLA at $800^{\circ} \mathrm{C}$ for $20 \mathrm{~ms}$ the PL measurements reveal only weak $\mathrm{B}-\mathrm{B}$ luminescence. This shows that the implanted region still contains many defects which cannot be detected by the Raman spectroscopy. Independent of preheating, the highest PL intensity was obtained from the samples annealed at $1200^{\circ} \mathrm{C}$ for $20 \mathrm{~ms}$.

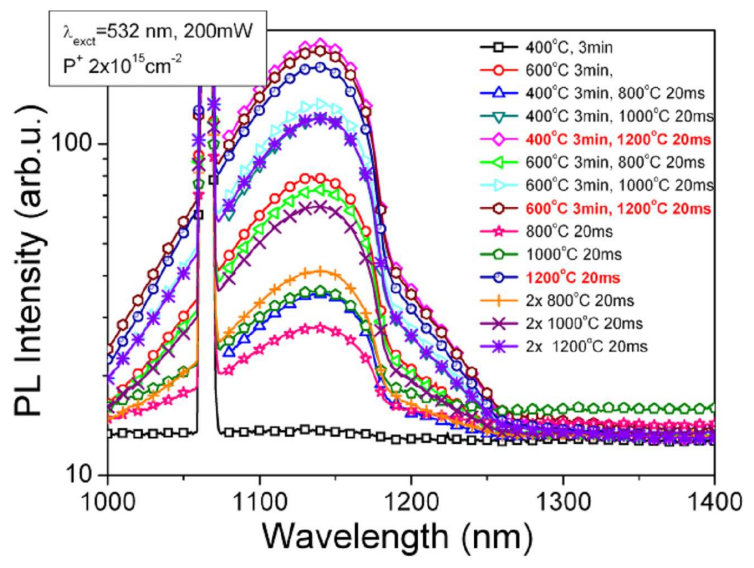

Fig. 3. Room temperature PL spectra obtained from crystalline silicon implanted with $\mathrm{P}^{+}$ions and annealed at different temperatures and time. 
After ion implantation and annealing the optical property of the wafer is homogeneous throughout the wafer area for the crystalline silicon but not for the multicrystalline silicon. The structural and optical properties of the mc-Si depend on the grain size, local crystallographic orientation, grain boundaries etc. In order to characterise the processing homogeneity (ion implantation, flash lamp annealing) the room temperature PL and Raman mapping was performed (see Fig. 4a and b, respectively).

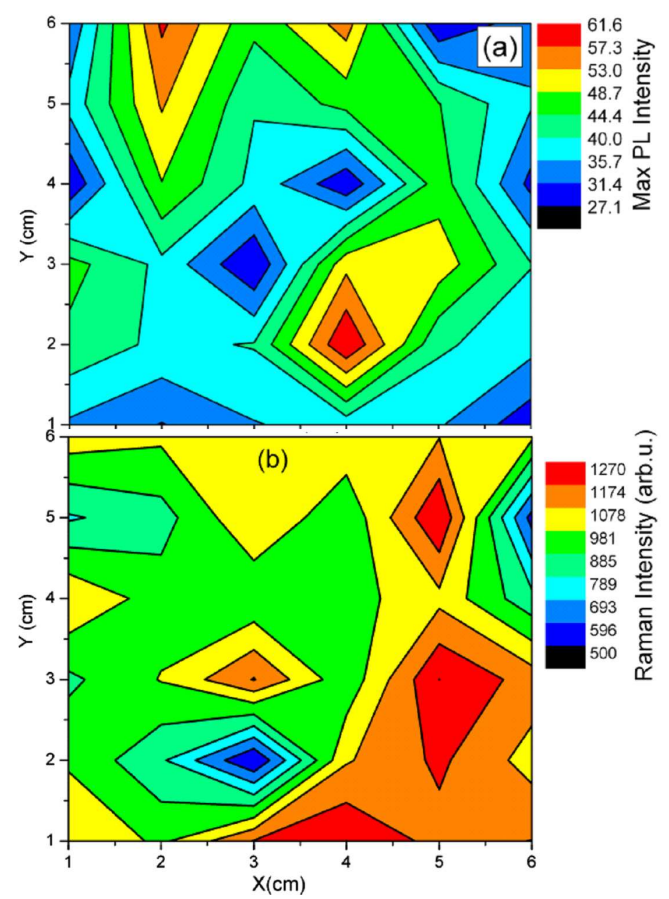

Fig. 4. Room temperature PL (a) and Raman (b) mapping of the multicrystalline silicon wafer.

The PL and Raman measurements were done on the $6 \times 6 \mathrm{~cm}^{2}$ wafers $\mathrm{P}^{+}$implanted and annealed at $1000^{\circ} \mathrm{C}$ for $20 \mathrm{~ms}$ with $400^{\circ} \mathrm{C}$ for $3 \mathrm{~min}$ preheating. In both cases the same laser light as the excitation source was used. The samples were measured in 36 points with $1 \mathrm{~cm}$ step. The maximum PL intensity is in the range of $40-50$ counts for $90 \%$ of the wafer area. As can be seen in Fig. 4a the inhomogeneity of the mc-Si wafer after solar cell processing is below $12 \%$. The same results were obtained according to the Raman spectroscopy (see Fig. 4b).

Figure 5 shows correlation between PL intensity and sheet resistance measured on the c-Si wafer. The SR strongly depends on the defects density and the concentration of phosphorus activated in silicon during annealing. The defects have also huge influence on the optical properties of the solar cell processed silicon wafer. Comparing the integrated PL intensity (B-B transition in silicon) and the results of the sheet resistance measurements, we have found that the PL intensity is inversely proportional to the SR value. The maximum PL intensity was obtained from the samples annealed at $1200^{\circ} \mathrm{C}$

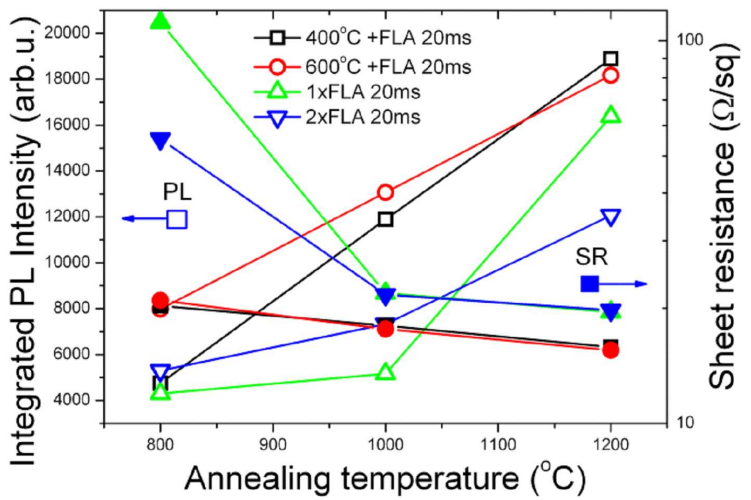

Fig. 5. Integrated PL intensity (left axis) and sheet resistance measurements as a function of the annealing temperature of the $\mathrm{P}^{+}$implanted and annealed c-Si wafer.

for $20 \mathrm{~ms}$ with $400^{\circ} \mathrm{C}$ preheating for $3 \mathrm{~min}$, at the same time the SR shows the minimum value $(16 \Omega / \mathrm{sq})$. It is worth noting that the acceptable value of the SR in the solar cell technology is in the range of $50 \Omega /$ sq. On the other hand, after low temperature flash lamp annealing or just after preheating the samples exhibit very poor optical properties and high resistivity.

\section{Conclusions}

We have shown that the high temperature and short term annealing process (FLA) is the most suitable for the large area solar cell production. The flash annealing at a temperature above $1000^{\circ} \mathrm{C}$ for $20 \mathrm{~ms}$ is sufficient to activate phosphorus atoms and recrystallize silicon layer damaged during ion implantation. Moreover, the tensile stress in the mc-Si can be easily removed during FLA. The Raman spectroscopy combined with PL measurements is a fast and non-destructive method for solar cell characterization.

\section{Acknowledgments}

This work was performed within the Cluster of Excellence "Structure Design of Novel High-Performance Materials via Atomic Design and Defect Engineering (ADDE)" that is financially supported by the European Union and by the Ministry of Science and Art of Saxony (SMWK).

\section{References}

[1] K. Ohdaira, T. Fujiwara, Y. Endo, K. Shiba, H. Takemoto, H. Matsumura, Jpn. J. Appl. Phys. 49 04DP04 (2010).

[2] H. Watanabe, H. Miki, S. Sugai, K. Kawasaki, T. Kioka, Jpn. J. Appl. Phys. 33, 4491 (1994).

[3] A. Suboundji, T. Mohammed-Brahim, G. Andrä, J. Bergmann, F. Falk, J. Non-Cryst. Solids 338-340, 758 (2004). 
[4] N.K. Mudugamuwa, A. Adikaari, D. Dissanayake, V. Stolojan, S. Silva, Sol. Energy Mater. Sol. Cell 92, 178 (2008).

[5] K. Sopian, N. Amin, N. Asim, S.H. Zaidi, Europ. J. Sci. Res. 24, 365 (2008).

[6] T. Kim, G. Kim, Y. Yoon, C. Kim, B. Lee, S. Yoo, Jpn. J. Appl. Phys. Part 1 39, 5775 (2000).

[7] F. Terai, S. Matunaka, A. Tauchi, Ch. Ichimura, T. Nagatomo, T. Homma, J. Electrochem. Soc. 153 H147 (2006).

[8] Ch.H. Poon, A. See, Y. Tan, M. Zhou, D. Gui, J. Electrochem. Soc. 155, H59 (2008).

[9] W. Anwand, S.Z. Xiong, C.Y. Wu, T. Gebel, Th. Schumann, G. Brauer, W. Skorupa, Acta Phys. Pol. A 113, 1273 (2008).

[10] J.E. Smith Jr, M.H. Brodsky, B.L. Crowder, M.I. Nathan, A. Pinchuk, Phys. Rev. Lett. 26, 642 (1971).
[11] Springer Handbook of Condensed Matter and Materials Data, Eds. W. Martienssen, H. Warlimont, Springer, Berlin 2005.

[12] J. Jin, Z. Yuan, L. Huang, S. Chen, W. Shi, Z. Cao, Q. Lou, Appl. Surf. Sci. 256, 3453 (2010).

[13] E. Bonera, M. Fanciulli, M. Mariani, Appl. Phys. Lett. 87, 111913 (2005).

[14] P. Lengsfeld, N.H. Nickel, Ch. Genzel, W. Fuths, J. Appl. Phys. 91, 9128 (2002).

[15] C. Georgi, M. Hecker, E. Zschech, J. Appl. Phys. 101, 123104 (2007). 